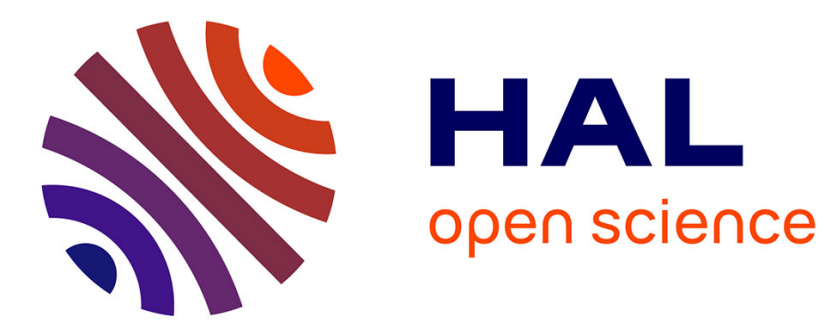

\title{
Freezing parameters of soft spheres
}

Nigel Wilding

\section{To cite this version:}

Nigel Wilding. Freezing parameters of soft spheres. Molecular Physics, 2009, 107 (04-06), pp.295-299. 10.1080/00268970802603507 . hal-00513238

\section{HAL Id: hal-00513238 \\ https://hal.science/hal-00513238}

Submitted on 1 Sep 2010

HAL is a multi-disciplinary open access archive for the deposit and dissemination of scientific research documents, whether they are published or not. The documents may come from teaching and research institutions in France or abroad, or from public or private research centers.
L'archive ouverte pluridisciplinaire HAL, est destinée au dépôt et à la diffusion de documents scientifiques de niveau recherche, publiés ou non, émanant des établissements d'enseignement et de recherche français ou étrangers, des laboratoires publics ou privés. 


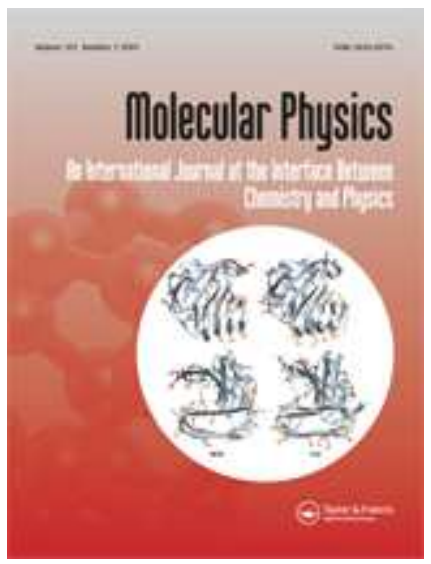

\section{Freezing parameters of soft spheres}

\begin{tabular}{|r|l|}
\hline Journal: & Molecular Physics \\
\hline Manuscript ID: & TMPH-2008-0240.R1 \\
\hline Manuscript Type: & Special Issue Paper - Dr. Jean-Jacques Weis \\
\hline Date Submitted by the \\
Author: & 23-Oct-2008 \\
\hline Keywords: & Phase transitions, Freezing, Molecular simulation \\
\hline & \\
\hline & Wilding, Nigel; University of Bath, Physics \\
\hline $\begin{array}{l}\text { Note: The following files were submitted by the author for peer review, but cannot be converted } \\
\text { to PDF. You must view these files (e.g. movies) online. }\end{array}$ \\
\hline \begin{tabular}{l} 
paper.tex \\
\hline
\end{tabular}
\end{tabular}

\section{S ScholaroNE \\ Manuscript Central}




\title{
Freezing parameters of soft spheres
}

\author{
Nigel B. Wilding \\ Department of Physics, University of Bath, Bath BA2 7AY, U.K.
}

\begin{abstract}
The phase switch Monte Carlo method is employed to determine the freezing properties of particles interacting via an $r^{-12}$ repulsive potential. We establish that at the equilibrium freezing point the face centred cubic (fcc) structure has a free energy that is lower than that of the metastable body centred cubic (bcc) structure by $0.0184(4) k_{B} T$ per particle. Thus the bcc structure is not the stable solid phase at freezing despite being commonly observed in simulations that physically nucleate the solid from the fluid. For the equilibrium fluid-fcc transition point we report accurate estimates of the coexistence pressure and densities for systems of sizes $N=108, N=256$ and $N=500$ particles.
\end{abstract}

\section{INTRODUCTION}

The task of constructing a complete phase diagram for a model system typically involves determining the loci of a variety of phase coexistence lines, notably the liquidvapor and fluid-solid lines. In practice there are a variety of classes of simulation scheme one can utilize to achieve this [1], but arguably the most effective approaches are those which, in the course of a single simulation run, sample the configuration spaces of both of the competing phases [2]. Doing so allows one to directly, accurately, and transparently compute the relative configurational weights of the two phases (ie. their free energy difference), and thence the coexistence parameters.

In the context of the vapor-liquid transition, such "direct" methods for determining coexistence parameters (and the associated critical behaviour) have been available for some years $[3,4]$ and are now routinely deployed. By contrast, progress in tackling fluid-solid coexistence has been somewhat slower, hampered by problems which stem from the distinct symmetries of the coexisting phases [5]. Recently, however, simulation techniques for dealing satisfactorily with fluid-solid transitions have begun to appear (see eg. refs. $[6,7]$ ). In this paper, we deploy one of them - the Phase Switch Monte Carlo method [5]- to study the freezing parameters of finite systems of repulsive soft spheres described by an $r^{-12}$ interaction potential.

Previous simulation work on the freezing transition of soft spheres has been reported by Hoover et al [8], who employed an indirect technique - thermodynamic integration - to estimate the coexistence parameters. Separate measurements of the free energies of the fluid phase and crystal phase were made for a range of near coexistence pressures, and then matched to locate the transition point. A different aspect of the freezing of soft spheres has been studied by Van Duijneveldt and Frenkel [9], who measured the free energy barriers to crystal nucleation. They observed that the barrier to the formation of body-centred-cubic (bcc) structures from the melt is considerably less than that for face-centred-cubic (fcc) crystallites (see also ref. [10]) which appear only later in the growth process. Other more recent studies have also reported the appearance of bcc structures in simulation of the freezing of soft spheres $[11,12]$. Such findings have been rationalized in terms of measurements of the fluid-bcc and fluid-fcc interfacial tensions [13] which find that the surface tension for bcc is less than that for fcc. Whilst all these workers emphasised that the appearance of bcc structures does not imply that bcc is the stable crystalline phase at melting - which is instead presumably fcc in character given its more efficient packing - we know of no direct measurements to corroborate this.

The structure of this short paper is as follows. We start by giving an outline of the phase switch Monte Carlo method which we have used to study the fluid-solid transitions for softly repulsive spheres. We then present accurate estimates of the coexistence pressure and density for the equilibrium fluid-fcc transition for systems of sizes $N=108, N=256$ and $N=500$ particles and discuss the finite-size effects in these estimates. Finally we provide a direct measurement of the difference in Gibbs free energies of fcc and bcc structures at the equilibrium freezing density, confirming that fcc is indeed the stable solid phase at the equilibrium freezing point.

\section{OUTLINE OF METHODOLOGY}

Phase Switch Monte Carlo (PSMC) is a general simulation scheme for sampling the disjoint configuration spaces associated with coexisting phases within a single simulation. The method employs biased sampling techniques to enhance the probabilities of certain "gateway" states in the configuration space of each phase which are such that a global switch to the other phase can be implemented. It permits direct and transparent determination of equilibrium coexistence parameters and their uncertainties, and the systematic quantification of finite-size effects.

The route PSMC takes between the coexisting phases is artificial and therefore provides no information on the physical dynamics of the phase change. However, for the task of determining coexisting parameters this can be a distinct advantage, particularly when one or both of the coexisting phases is crystalline. This is because simulation approaches which seek to emulate the physical nucleation process commonly encounter significant problems, principally a large degree of metastability of the phases, extended timescales for crystallization and a tendency for the crystals formed to exhibit defects. 
The PSMC method takes as its starting point the specification of a reference configuration $\{\vec{R}\}^{\gamma}$ for each of the phases (labelled $\gamma$ ) coexisting at the phase boundary. The specific choice of $\{\vec{R}\}^{\gamma}$ is arbitrary, the only condition being that it should be a member of the set of pure phase configurations identifiable as "belonging" to phase $\gamma$. For a crystalline phase, a suitably simple choice of $\{\vec{R}\}^{\gamma}$ is the set of lattice sites. For a fluid, a suitable choice is a randomly chosen fluid configuration.

The next step is express the coordinates of each particle in phase $\gamma$ in terms of the displacement from its reference site, i.e.

$$
\vec{r}_{i}^{\gamma}=\overrightarrow{R_{i}^{\gamma}}+\overrightarrow{u_{i}}
$$

Now, for displacement vectors that are sufficiently small in magnitude, one can clearly reversibly map any configuration $\{\vec{r}\}^{\gamma}$ of phase $\gamma$ onto a configuration of another phase $\tilde{\gamma}$ simply by switching the set of reference sites $\{\vec{R}\}^{\gamma} \rightarrow\{\vec{R}\}^{\tilde{\gamma}}$, while holding the set of displacements $\{\vec{u}\}$ constant. This switch, which forms the heart of the method, can be incorporated in a global MC move (see fig. 1).

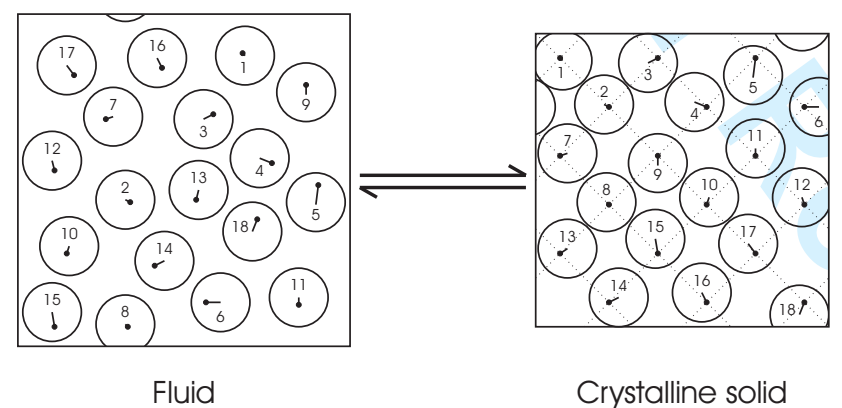

FIG. 1: Schematic illustration of the phase switch mechanism for the fluid and crystalline solid. The dots identify the representative sites $\{\vec{R}\}^{\gamma}$ in each phase; the displacement vectors $\{\vec{u}\}$ connect the centers of the distinguishable (numbered) particles to these sites. The switch operation shown swaps the representative sites of one phase for those of the other phase, whilst maintaining $\{\vec{u}\}$ constant. The particular configuration $\{\vec{u}\}$ shown is a "gateway" state (see text) because the magnitude of the effective energy change under the switch is small.

A complication arises however, because the displacements $\{\vec{u}\}$ typical for phase $\gamma$ will not, in general, be typical for phase $\tilde{\gamma}$. Thus the switch operation will mainly propose high energy configurations of phase $\tilde{\gamma}$ which are unlikely to be accepted as a Metropolis update. This problem can be circumvented by employing extended sampling (biasing) techniques to seek out those displacements $\{\vec{u}\}$ for which the switch operation is energetically favorable. These are the gateway configurations and typically correspond to displacement vectors which are small in magnitude. Fig. 2 shows a schematic representation of the procedure.

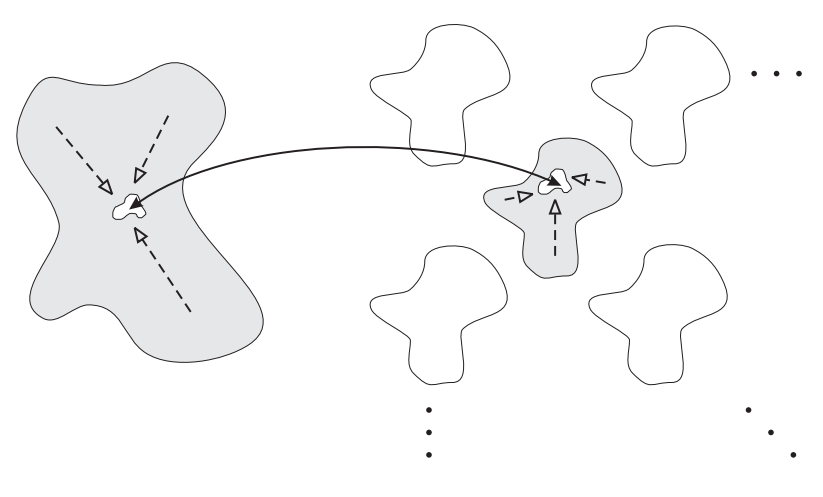

Fluid

FIG. 2: Schematic illustration of the phase switch operation in terms of the regions of configuration space associated with the fluid and crystalline solid phases. A bias (dashed arrows) is constructed such as to enhance the probability of the subsets of "gateway" states (the white islands) within the single-phase regions, from which the switch operation (the large arrow) will be accepted. Note that the switch accesses only one of the crystalline phase space replica fragments associated with permutations of particles amongst lattice sites [6].

The requisite bias is administered with respect to an "order parameter". This macrovariable is defined such that the set of typical microstates (particle configurations) associated with its range, form a continuous path in phase space which links the configurations of high statistical weight to the gateway configurations. Once a suitable biasing function has been determined to allow the system to reach the gateway states and hence switch (back and forth) from fluid to solid, a long run can be performed to accumulate statistics are on the relative configurational weights of the phases and their physical properties. The effects of the imposed bias are unfolded from the results in the standard manner [2] and statistical errors are obtained straightforwardly from the statistics of the inter-phase switch. Use of histogram reweighting [14] permits exploration of values of the temperature and pressure in the neighbourhood of a given simulation state point and serves as an invaluable aid for pinpointing coexistence parameters.

The statistical mechanical background to PSMC and extensive details of its implementation within a constant$N p T$ ensemble framework for problems of fluid-solid coexistence have been recently described elsewhere (see ref. [6]) and shall not therefore be repeated here. Instead we shall concentrate on the results for a system of soft spheres, as detailed below. 


\section{MODEL AND RESULTS}

The model we consider is the $r^{-12}$-soft-sphere potential,

$$
v(r)=\epsilon(\sigma / r)^{12} .
$$

The dimensionless density follows as $\rho^{\star}=\rho \sigma^{3}$, the dimensionless pressure as $p^{\star}=p \sigma^{3} / \epsilon$ and the dimensionless temperature as $T^{\star}=k_{B} T / \epsilon$. In fact, for this model the thermodynamic state is completely specified by a single dimensionless density parameter, namely

$$
\rho^{\prime}=\frac{N \sigma^{3}}{V}\left(\frac{\epsilon}{k_{B} T}\right)^{1 / 4} .
$$

Thus, the coexistence points for various temperatures scale exactly onto one another [8] and one therefore only needs to determine a single coexistence point. Accordingly in this work we shall consider the case $T^{\star}=1$.

In our constant- $N p T$ ensemble simulations, the interparticle potential was truncated at half the box size and periodic boundary conditions are applied. Since for the system sizes studied the value of the potential is extremely small at the typical cutoff radius, no correction was applied for the truncation.
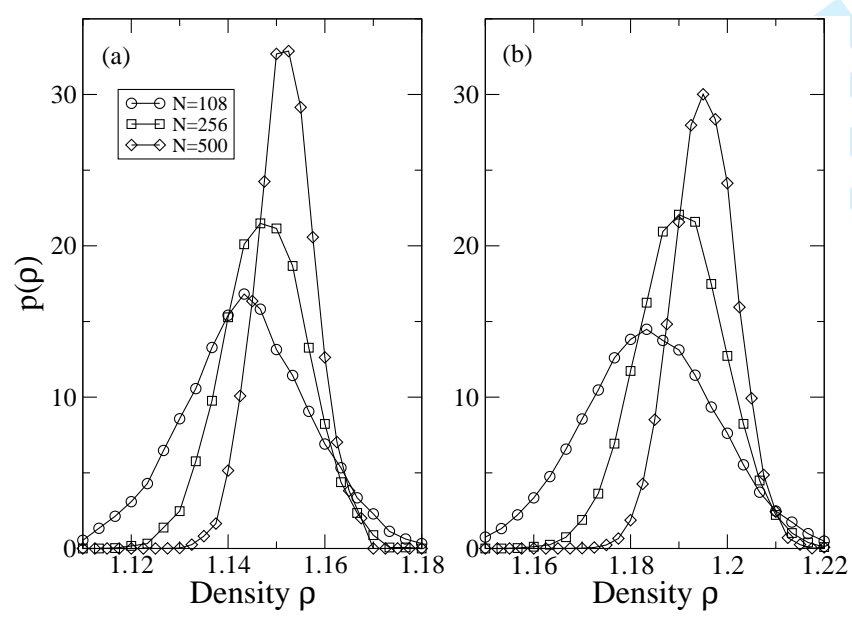

FIG. 3: The form of the density distribution $P(\rho)$ at fluid-fcc coexistence for the three system sizes indicated. (a): fluid phase distribution; (b) fcc solid phase distribution. The temperature was set to $T^{\star}=1$ in each case. The corresponding estimates of the coexistence parameters appear in Table I

We first consider the coexistence parameters for fluidfcc coexistence. The coexistence pressure is estimated as that pressure for which the system is found with equal probability in each of the two phases $[6,15]$. The resulting coexistence forms for the fluctuating density are shown in fig. 3 for systems of $N=108,256$ and 500 particles. Our numerical estimates of the coexistence parameters are summarized in Table I.

\begin{tabular}{c|ccc}
$N$ & $p^{\star}$ & $\rho_{f}^{\star}$ & $\rho_{s}^{\star}$ \\
\hline 108 & $21.84(5)$ & $1.143(12)$ & $1.184(13)$ \\
256 & $22.32(3)$ & $1.148(9)$ & $1.190(9)$ \\
500 & $22.66(1)$ & $1.152(6)$ & $1.195(6)$
\end{tabular}

TABLE I: Measured coexistence parameters for the fluid-fcc transition for the three system sizes studied. $\rho_{f}^{\star}$ and $\rho_{s}^{\star}$ are the fluid and solid phase number densities respectively. The statistical errors indicated correspond to one standard deviation.

Ideally, one would like to be able to extrapolate these estimates of coexistence parameters for finite-sized systems to the thermodynamic limit. Unfortunately at present there is no rigorous finite-size scaling theory for fluid-solid coexistence, the finite-size properties of which seem to differ from those of fluid-fluid coexistence. For hard sphere systems, independent PSMC studies of the freezing parameters revealed a clear $1 / N$ scaling of the coexistence pressure $[5,16]$ (see fig 4(a)). By contrast in the present model of soft spheres, the case for such a scaling is numerically unconvincing (see fig $4(\mathrm{~b})$ ) since a $1 / N$ fit lies well outside our error bars, the form of the deviation suggesting a weaker than $1 / N$ convergence. A similar finding applies to the estimates of the coexistence densities. We return to this point below.
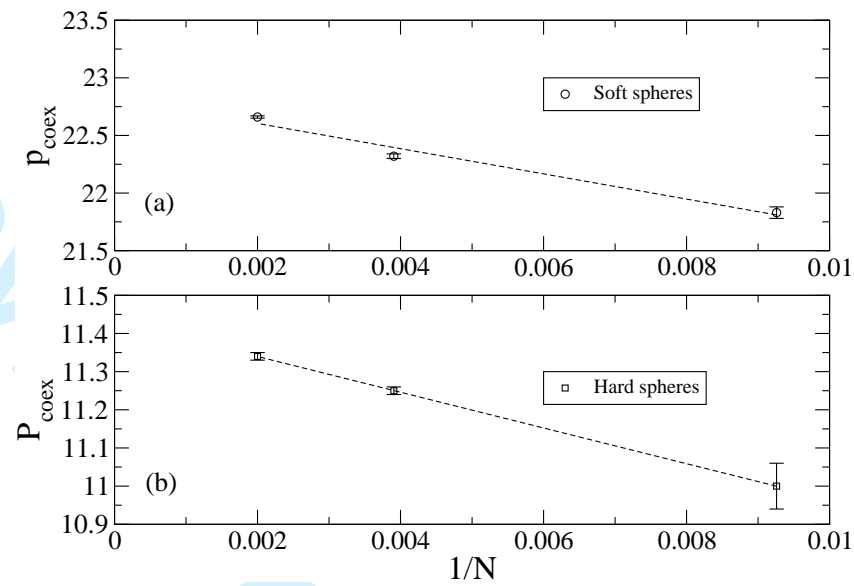

FIG. 4: (a) Measured coexistence pressures for the soft sphere system, for the three system sizes $N=108,256$ and 500, plotted as a function of $1 / N$. (b) Corresponding plot for hard spheres using the results from Errington's PSMC study of the same system sizes [16].

Our results can be compared with those of Hoover et al. [8] who performed thermodynamic integration to obtain the free energy of the fcc and fluid phases of soft spheres for a range of densities. For a system of $N=500$ particles, they quote coexistence parameters of $p^{\star}=22.56(40) \rho_{f}^{\star}=1.150(8), \rho_{s}^{\star}=1.194(8)$, estimates which are clearly in good accord with our own (Table. I). Davidchack and Laird also report estimates for the coexistence parameters of soft spheres, obtained via constant NVT Molecular dynamics simulations of large sys- 
tems $(N \approx 15000)$ containing an interface. They quote $p^{\star}=23.41(4), \rho_{f}^{\star}=1.163(1), \rho_{s}^{\star}=1.206(1)$. It seem difficult to compare these results with our findings given the disparity in system sizes and the lack of a rigorous finite-size scaling theory.

Finally, we have performed phase switch measurements of the relative free energy of fluid and bcc phases for a system of $N=250$ soft spheres. The bcc phase is (meta)stable on the timescale of our simulations allowing us to measure its Gibbs free energy relative to the fluid. We find that at the pressure $p^{\star}=22.32(2)$ for which the $N=256$ particle system exhibits fluid-fcc coexistence, the bcc solid is less stable than the fluid (and hence too the fcc solid) by $0.0184(4) k_{B} T$ per particle. We have also estimated the pressure for which the metastable bcc structure coexists with the fluid for $N=250$. This occurs at $p^{\star}=23.10(5)-$ considerably higher than the true freezing pressure.

\section{CONCLUSIONS}

In this paper we have verified that the soft sphere fluid does indeed freeze to the expected fcc structure. At the equilibrium freezing point, the competing bcc structure was found to be metastable allowing us to measure the difference in free energy to the fcc structure. We find that the free energy per particle of the bcc structure exceeds that of the fcc structure by $0.0184(5) k_{B} T$ per particle. Pseudo coexistence between the fluid and the metastable bcc structure occurs at a pressure some $3.5 \%$ above the true freezing point.

We have also reported accurate fluid-fcc coexistence parameters for three system sizes of soft spheres. In contrast to hard sphere systems of the same system sizes (studied by the same technique), soft spheres seem to show a weaker than $1 / N$ convergence of the coexistence parameters although the range of system sizes on which this finding is based is admittedly limited. Interestingly, a finite-size convergence that is weaker than $1 / N$ was also reported in PSMC studies of the Lennard-Jones system $[6,16]$. In the latter works the deviations from $1 / N$ scaling were tentatively ascribed to the effects of the system size on the truncation of the long ranged attractive part of the LJ potential. The finding of the present work would suggest that there may be more subtle differences between finite-size effects for freezing of hard and soft particles. While the reasons for such difference are not clear to us at present, the matter certainly warrants further theoretical and numerical attention.
1] D. Frenkel and B. Smit, Understanding Molecular Simulation (Academic, San Diego, 2002).

[2] A.D. Bruce and N.B. Wilding, Phys. Chem. Chem. Phys 127, 1 (2003).

[3] A.Z. Panagiotopoulos, Mol. Phys. 61, 813 (1987).

[4] N.B. Wilding, Phys. Rev. E 52, 602 (1995).

[5] N.B. Wilding and A.D Bruce, Phys. Rev. Lett. 85, 5138 (2000).

[6] G.C. McNeil-Watson and N.B. Wilding, J. Chem. Phys. 124, 064504 (2006).

[7] D.M. Eike, J.F. Brennecke, and E. J. Maginn, J. Chem. Phys. 122, 014115 (2005).

[8] W.G. Hoover et al., J. Chem. Phys. 52, 4931 (1970).

[9] J.S. van Duijneveldt and D. Frenkel, J. Chem. Phys. 96, 4655 (1992).
[10] M.J. Ruiz-Montero P.R. ten Wolde and D. Frenkel, Phys. Rev. Lett. 75, 2714 (1995).

[11] L.A. Fernández, V. Martín-Mayor, and P. Verrocchio, Phys. Rev. Lett. 98, 085702 (2007).

[12] M. Chopra, M. Müller, and J.J. de Pablo, J. Chem. Phys. 124, 134102 (2006).

[13] R.L. Davidchack and B.B. Laird, Phys. Rev. Lett. 94, 086102 (2005)

[14] A.M. Ferrenberg and R.H. Swendsen, Phys. Rev. Lett. 63, 1195 (1989).

[15] M. Buzzacchi, P. Sollich, N.B. Wilding, and M. Müller, Phys. Rev. E. 73, 046110 (2006).

[16] J.R. Errington, J. Chem. Phys. 120, 3030 (2004). 


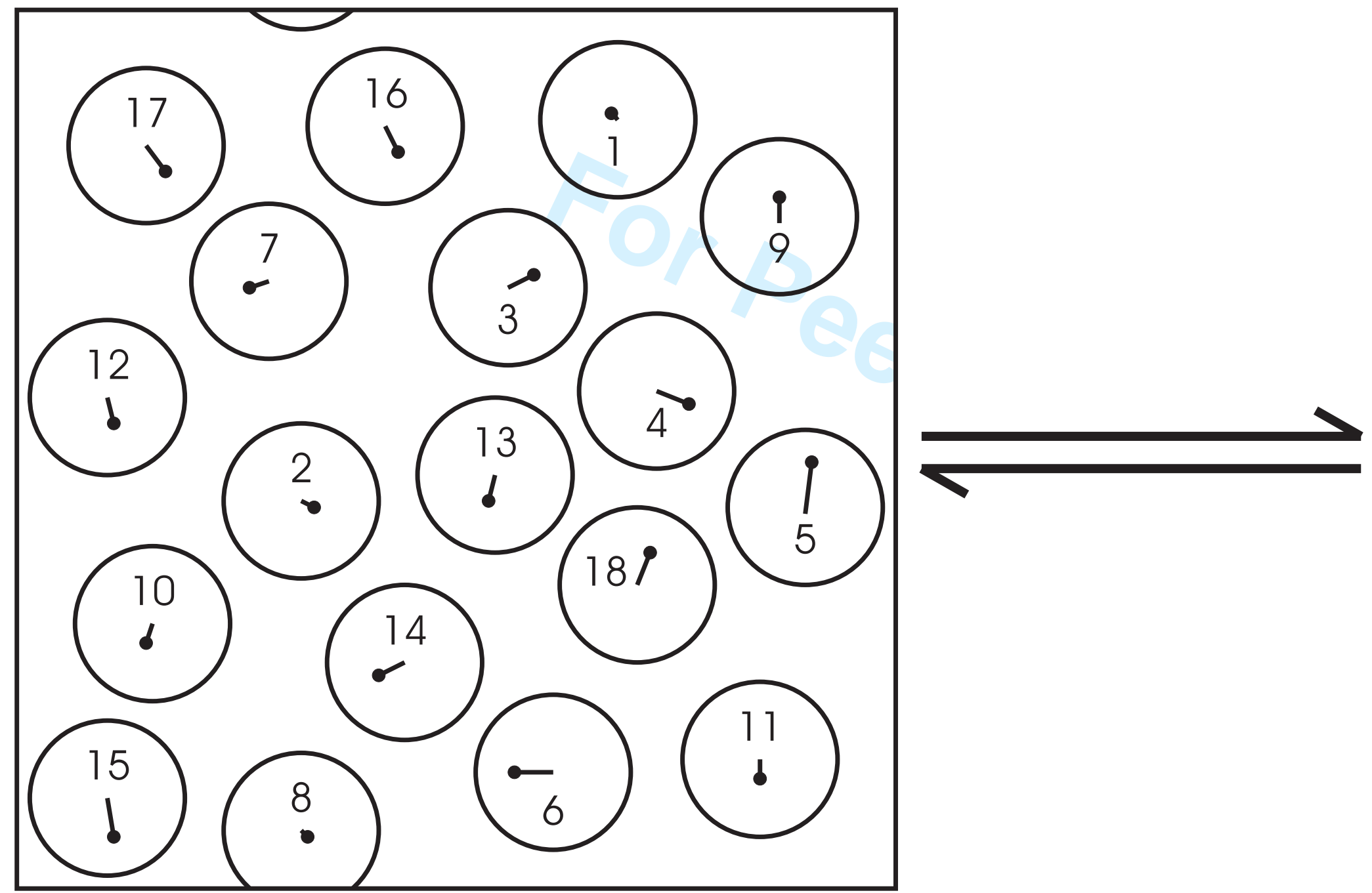

Fluid

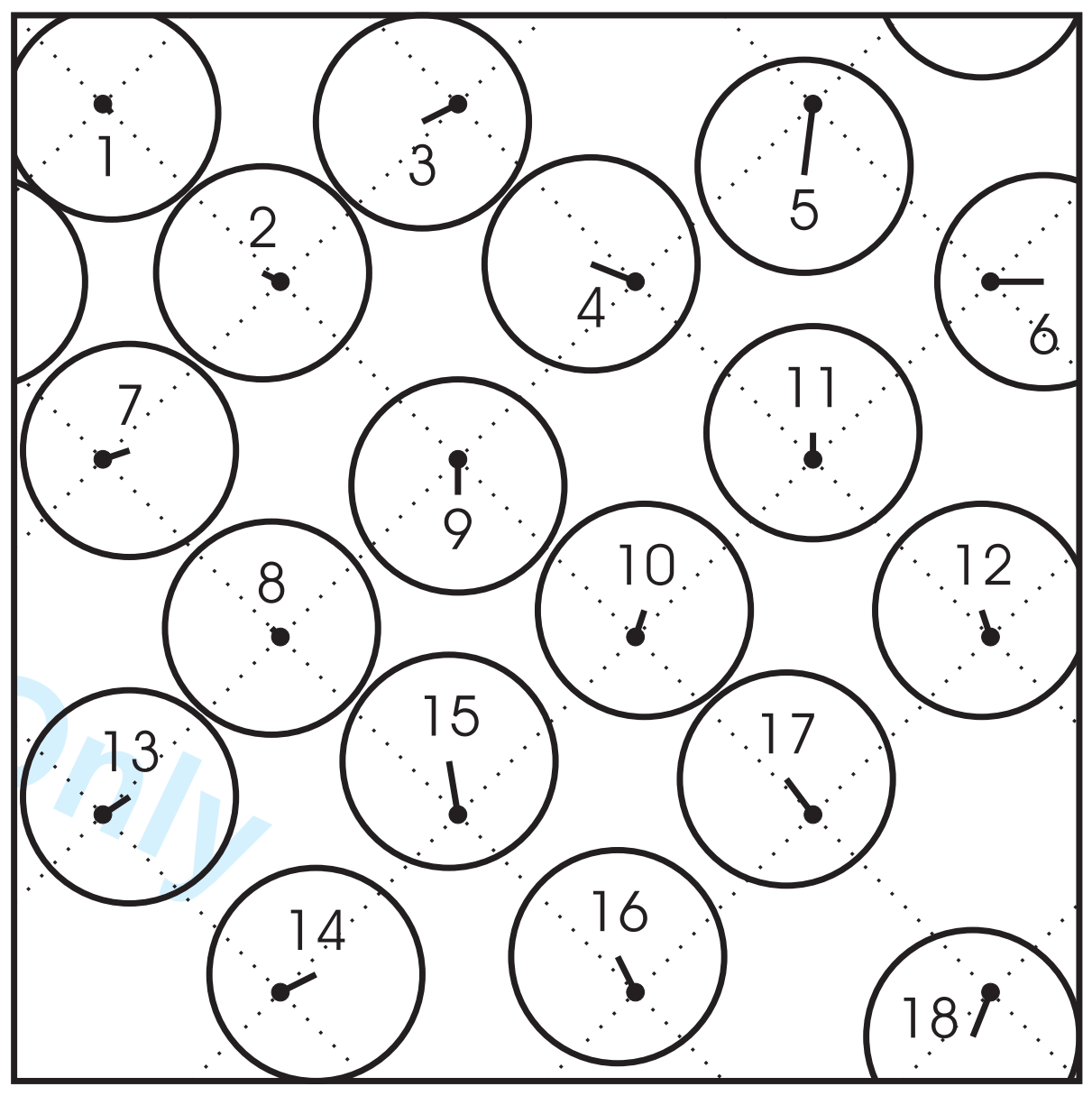

Crystalline solid 


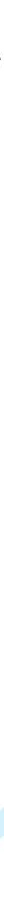



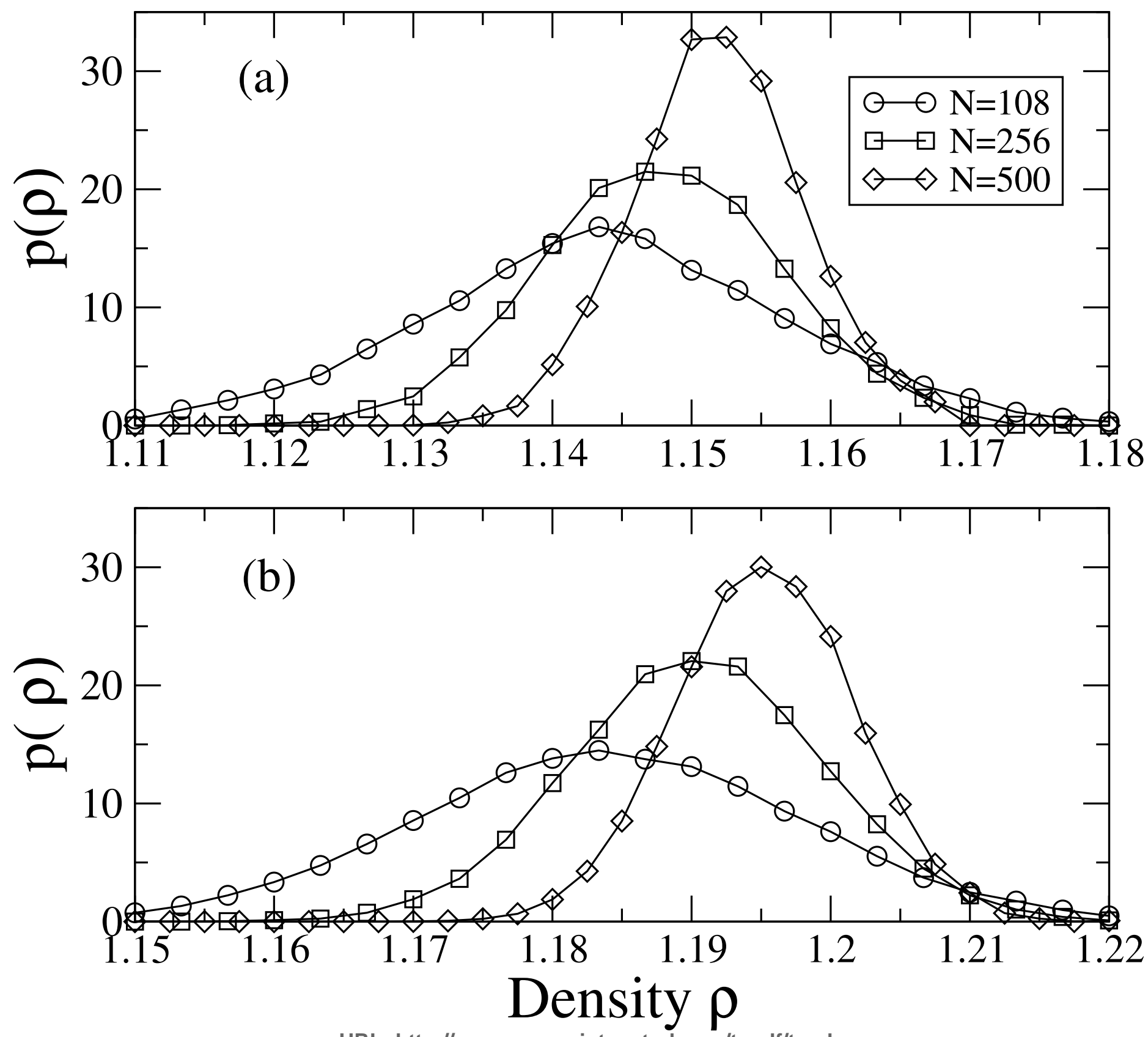

URL: http://mc.manuscriptcentral.com/tandf/tmph 

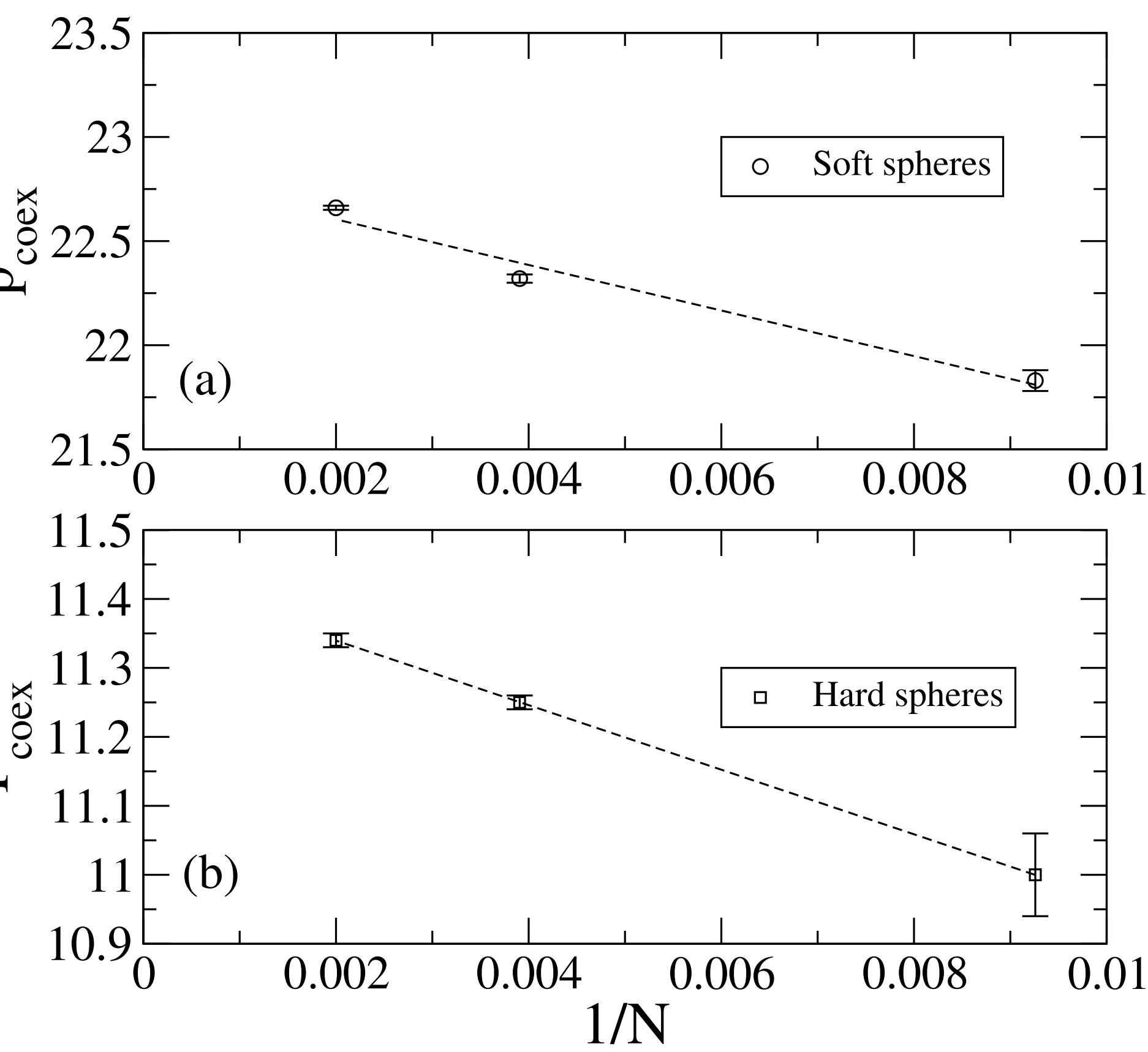

URL: http://mc.manuscriptcentral.com/tandf/tmph 